\title{
A 6DOF Flight Simulation Environment for General Aviation Aircraft with Control Loading Reproduction
}

\author{
Domenico P. Coiro* $\quad$ Agostino De $\mathrm{Marco}^{\dagger}$ \\ Fabrizio Nicolosi $i^{\ddagger}$ \\ Università degli Studi di Napoli "Federico II" - Dipartimento di Ingegneria Aerospaziale \\ Via Claudio, 2180125 Napoli, Italy
}

This paper presents the main features of a six-degree-of-freedom (6DOF) flight simulation laboratory operated by the authors at the University of Naples.

The aim of the flight simulator is twofold: serving as a research tool for model characterization and for the investigation of flying qualities of very-light and ultra-light aircraft; and offering a training options to the pilots of such airplanes. For these reasons the simulator cockpit has been conceived and set up as a generic cabin of a general aviation aircraft.

The software suite that guides the various components of the system is based mainly on the features of FlightGear, an open-source flight simulation software. The simulation of aircraft motion, the cockpit instrument panel and flight controls, the outside scenery are all managed by a number of instances of FlightGear. All FlightGear instances are appropriately executed on different machines and communicate with each other via net protocols.

Simulations are also supported by two other software modules. The first one controls the 6DOF motion of the cockpit. The second module implements a force reproduction system on the cockpit controls. An overview of all these modules is given in the paper, along with the discussion of the advantages and potentialities given by the source code accessibility and the high configurability of FlightGear.

The force feedback model is particularly important to the purposes that this flight simulation facility has being designed for. To obtain an enhanced realism in piloting efforts, particular care has been taken to implement hinge moment equations in the simulation software. The result is a reliable closed-loop force-feedback system on all aircraft commands. Two useful and noteworthy generalizations have been implemented in this context: the effect of the mechanical linkage dynamics on the control surface motion and the effects on the control displacement due to the mechanical friction and to the presence of springs. The geometric, mass, inertia characteristics of each control surface and the hinge moment coefficients are managed by the control loading software. All the details of this model are given in the paper.

\section{Introduction}

The authors of this paper have worked to the specifications, the development and the final acceptance procedure of a recently acquired simulation facility. The whole system has been designed to be operated both as a driving simulator and as a flight simulator and is going to be managed by two different research teams, one including the authors, the other coming from the Transportation Department of the same University.

The system is a full scale simulator and includes real vehicle mock-up, a 6DOF motion system, a large projection system, and a force feedback module. The half-body of a real car and the aircraft cockpit mock-up are exchangeable and easily installed on the motion platform. Apart from those characteristics shared with the automotive simulator team, the authors have worked mainly on the flight simulation side.

*Professor of Flight Mechanics, University of Naples Federico II, Department of Aerospace Engineering, coiro@unina.it.

$\dagger$ Assistant Professor, University of Naples Federico II, Department of Aerospace Engineering, agostino.demarco@unina.it.

¥Assistant Professor, University of Naples Federico II, Department of Aerospace Engineering, fabrizio.nicolosi@unina.it. 
The flight simulator cockpit has been conceived as a generic cabin of a small aircraft because the principal aim of this facility is to make investigations on flying qualities of light and ultra-light aircraft and to offer a training options to the pilots of such airplanes. In addition this simulation facility is used as an enhanced tool in aircraft stability and control teaching.

\section{Simulator layout and components}

The simulator is installed in Naples in one of the buildings belonging to Consiglio Nazionale delle Ricerche (National Research Council, CNR), Istituto Motori. The simulator room layout is shown in Figure 1.



Figure 1. Simulator room layout

As seen from the figure, the building is divided into three areas: the simulator main room, the supervisor room, the briefing room. The top-view shows the horizontal motion envelope of the cockpit, the main room dimensions, and other minor details. Also shown are the traces of hree large screens located in front of the cabin where three DLP projectors (DS30 from Christie Digital; 3000 lumens, $1280 \times 1024$, SXVGA) project a composite image of the virtual outside environment. 
This particular projection system, Figure 2, is preferred in car simulators and proved to be effective in the flight simulator presented here. The reproduced image corresponds to a $190^{\circ}$ field of view (horizontal).

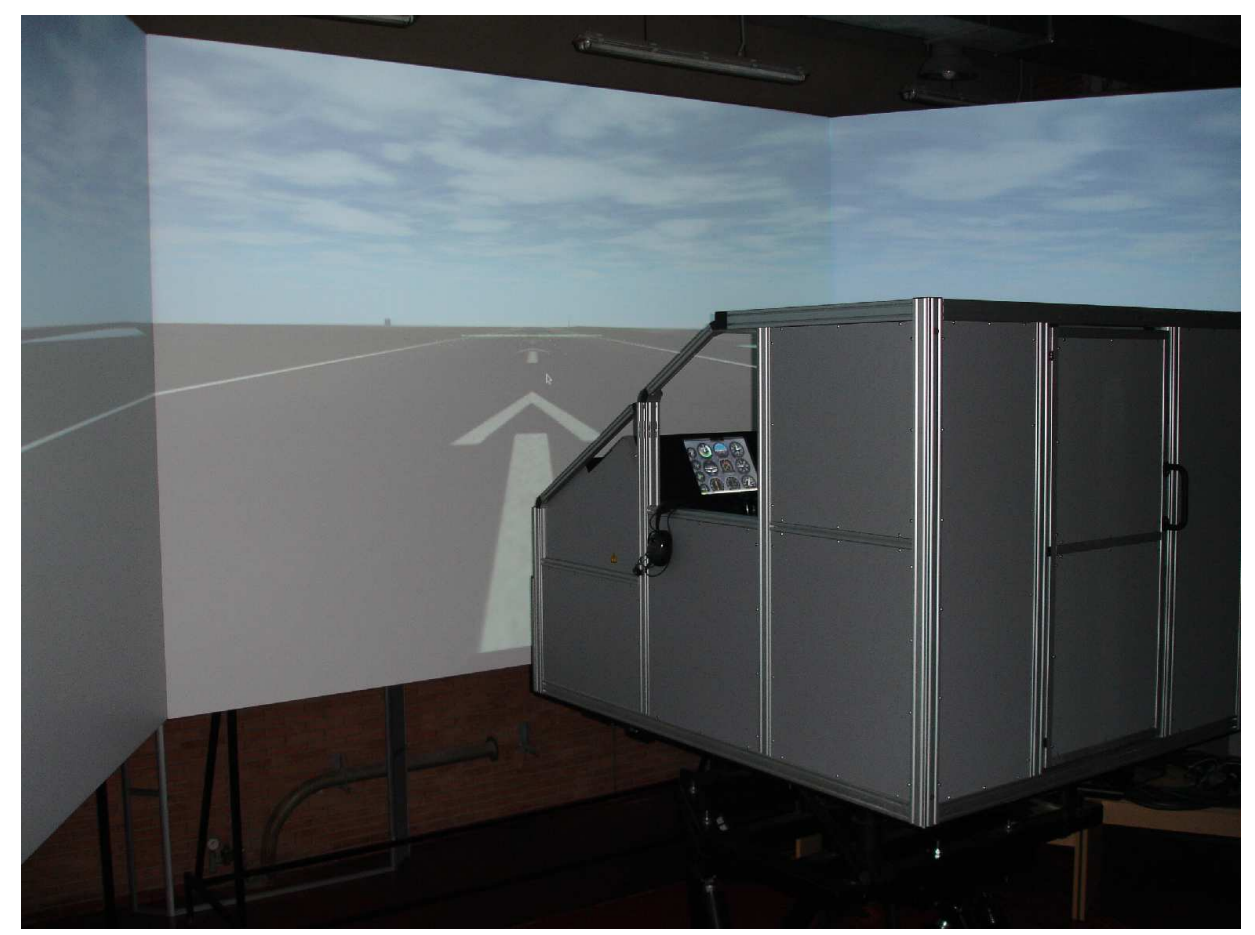

Figure 2. Projected images in front of the moving cabin during simulation

The subject pilot positioned in the cabin is given a motion cue during the simulation. The cue is obtained by animating the airplane mock-up with a six-degree-of-freedom motion platform, see Figures 3 and 4 .

Two available cockpits can be plugged on the motion platform and the simulator can be used both for driving simulation and flight simulation. To this aim a primary adaptor plate is fixed to the motion system. Each cockpit is mounted on a secondary adaptor plate, which is designed to be firmly coupled with the primary one. The primary plate is shown in Figure 3, on the top of the motion platform. The car and aircraft cockpits are swapped using a fork-lift truck. The entire operation takes less than 30 minutes. The cockpit in use is safely plugged on the motion system adaptor plate shown in the same figure. The mock-up of the aircraft cockpit is shown in Figure 4. 


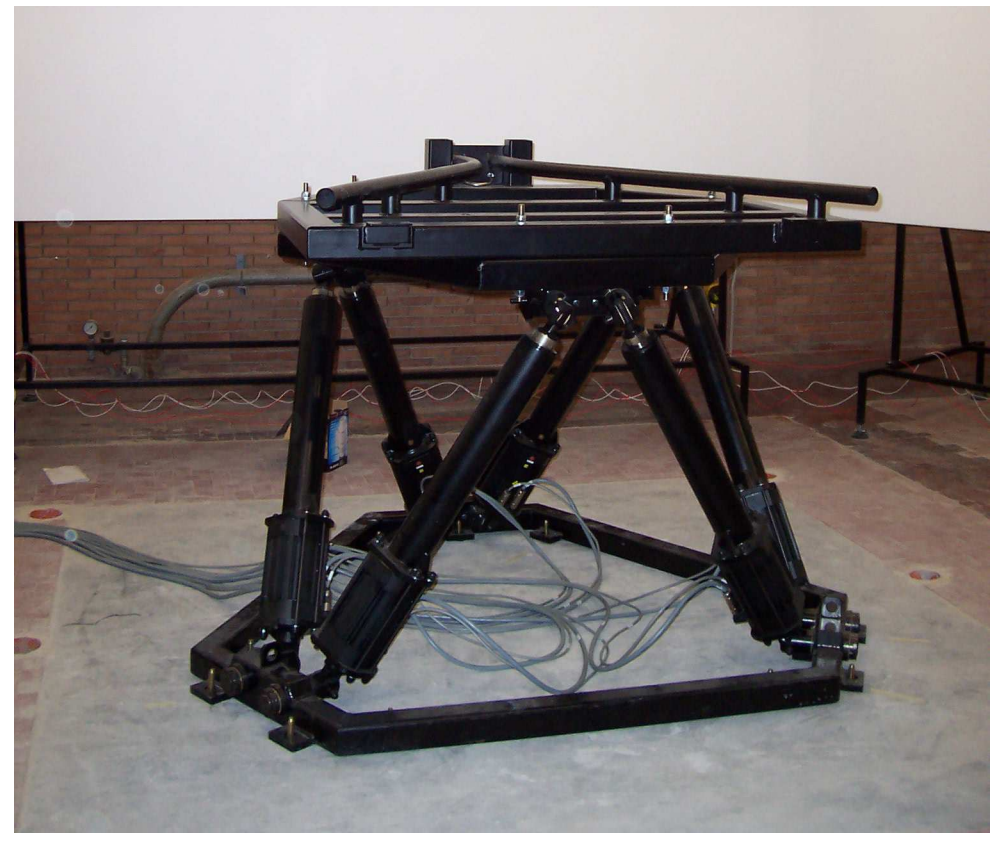

Figure 3. Motion system adaptor plate mounted on the top of the motion platform

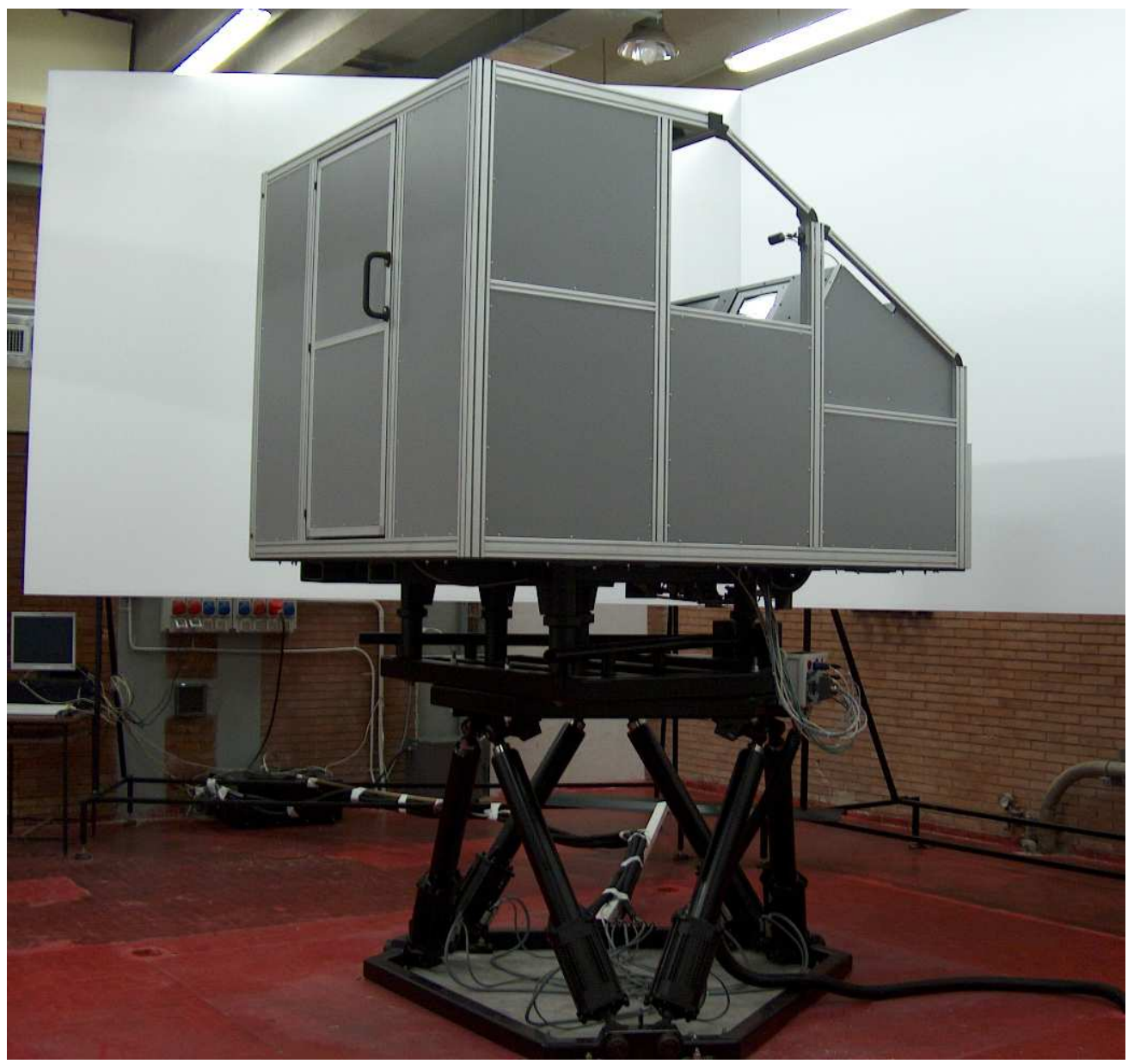

Figure 4. Mock-up of the aircraft cockpit mounted onto the motion platform 


\section{Mode of operation}

The effective use of the simulator requires the presence of an operator in the supervisor room, who is in charge and responsible of ensuring the safe use of the system. An emergency stop button in this room enables the supervisor to safely stop the simulation in case of emergency. The simulator room access doors are equipped with electrical contacts to ensure that they are closed and a red/green light is set outside the simulator room to prevent anyone to enter the room when the simulator is running.

The supervisor has a direct sight on the simulator area, as seen from Figure 5, and can stop the simulation if the safety conditions are broken.

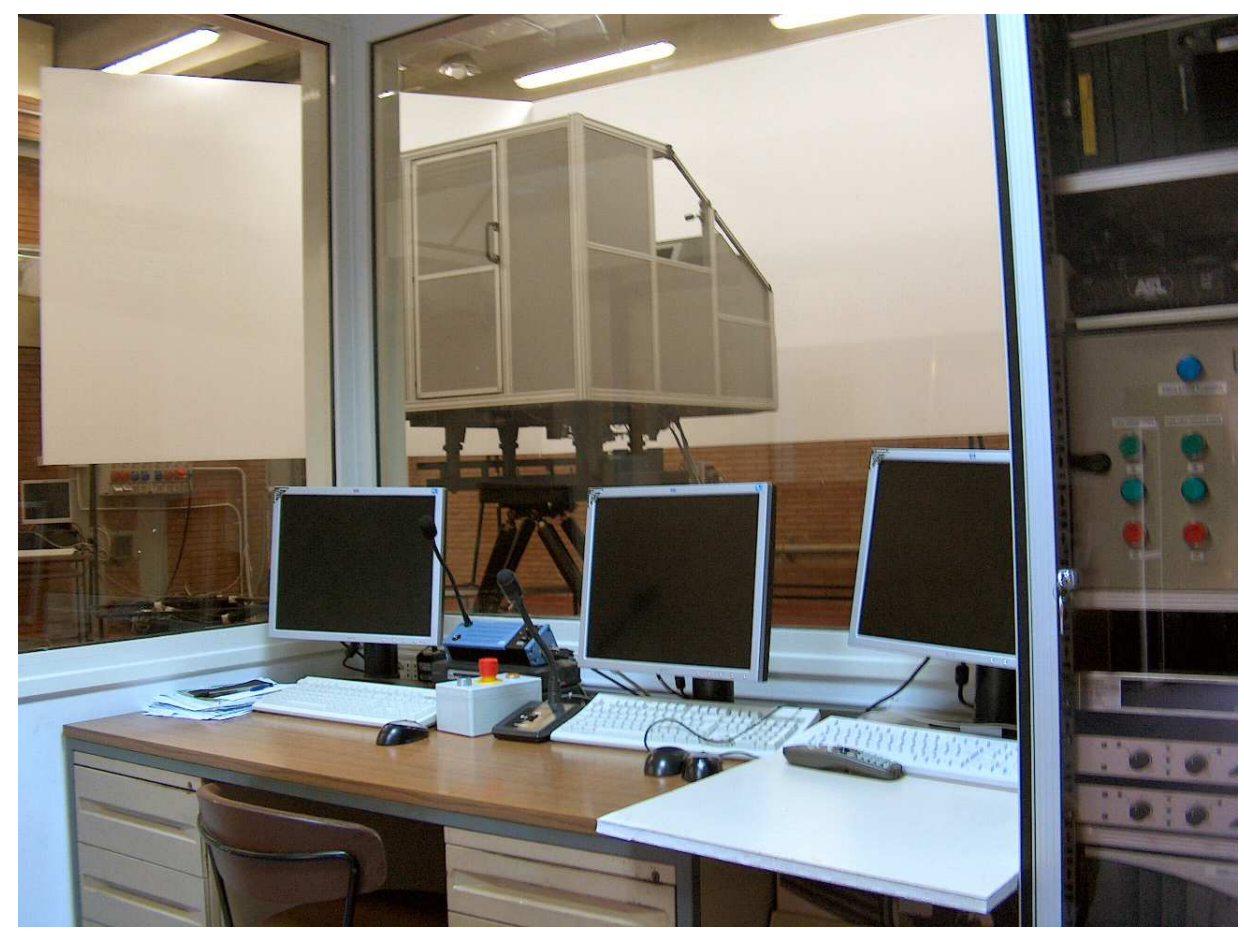

Figure 5. View from the supervisor room

The pilot inside the cabin is protected by electrical contacts on the cockpits doors, that have to be closed during the simulation, by a safety belt that has to be locked during the simulation, and by an emergency stop button onto the dashboard. A camera (audio/video) is installed inside the cockpit to observe the subject pilot and ear the sound within the cockpit from the supervisor room. The simulator motion area is protected to prevent anyone or any object to interfere with the simulator. The motion area is painted on the ground. The access stairs are linked to electrical contacts when they are at their parking places. These contacts are taken into account by the system.

\section{General architecture}

The flight simulator cockpit reproduces a generic cabin of a small aircraft, Figure 4 . The cockpit structure consists in an aluminum skeleton in which synthetic material (such as polycarbonate) sheets are slipped into the bones of the skeleton by means of appropriately designed slides. This solution has proved to be more convenient than having a real aircraft cockpit, both in terms of cost savings and of simulator usability. Inside the cockpit, the main instrument panel consists in two tactile LCD screens. One screen is used to display a virtual flight panel. The second screen enables the display of what is needed by the experiments, such as moving maps or real-time plots of flight parameter. A space is also reserved for a third screen. A picture of the dashboard with the two screens in use is shown in Figure 6.

The flight controls consist in: a Cirrus II Flight Console from Precision Flight Inc., a yoke, which is included in the original flight console but whose position has been conveniently modified, and a pair of rudder pedals. A picture of the controls is shown in fig. 7 . 


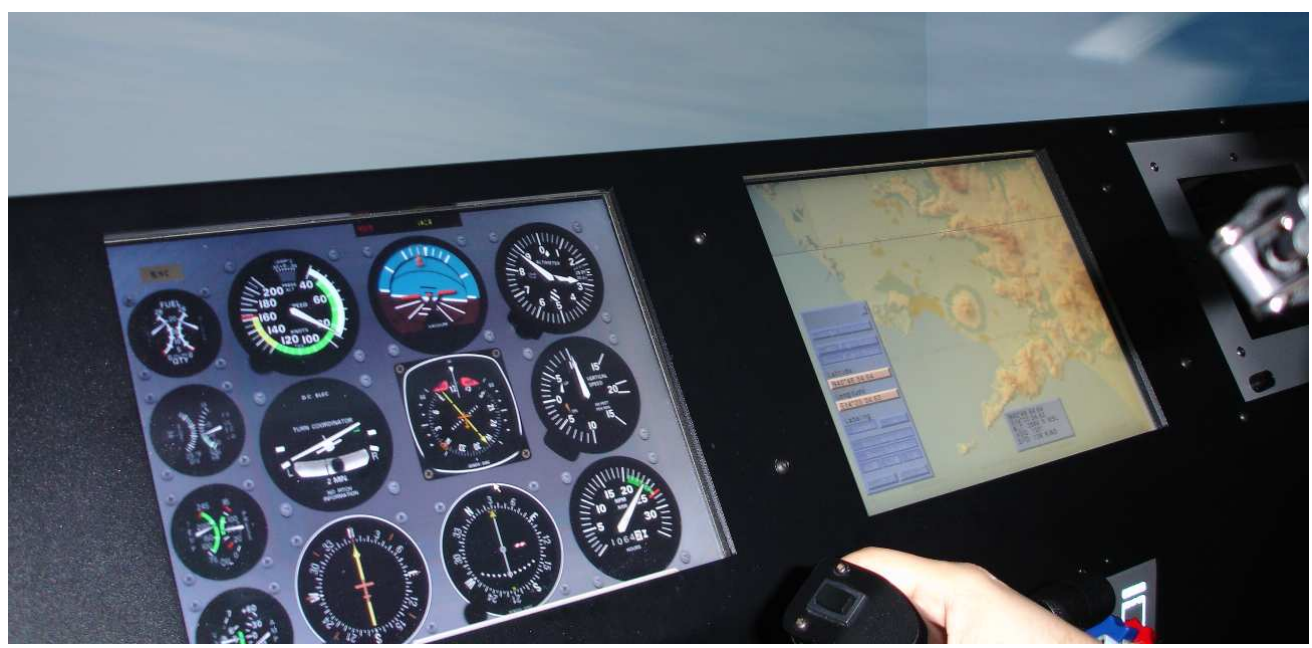

Figure 6. Virtual indicators and moving map inside the aircraft cockpit

To simulate the ATC radio link, the subject pilot has a headset with a microphone.

The yoke and the rudder pedals are equipped with a force feedback system giving to the subject an additional cue on the piloting effort. Figure 8 shows a detail (control panel back view) of the mechanical linkage connecting the yoke to one of the actuators of the force feedback system. 


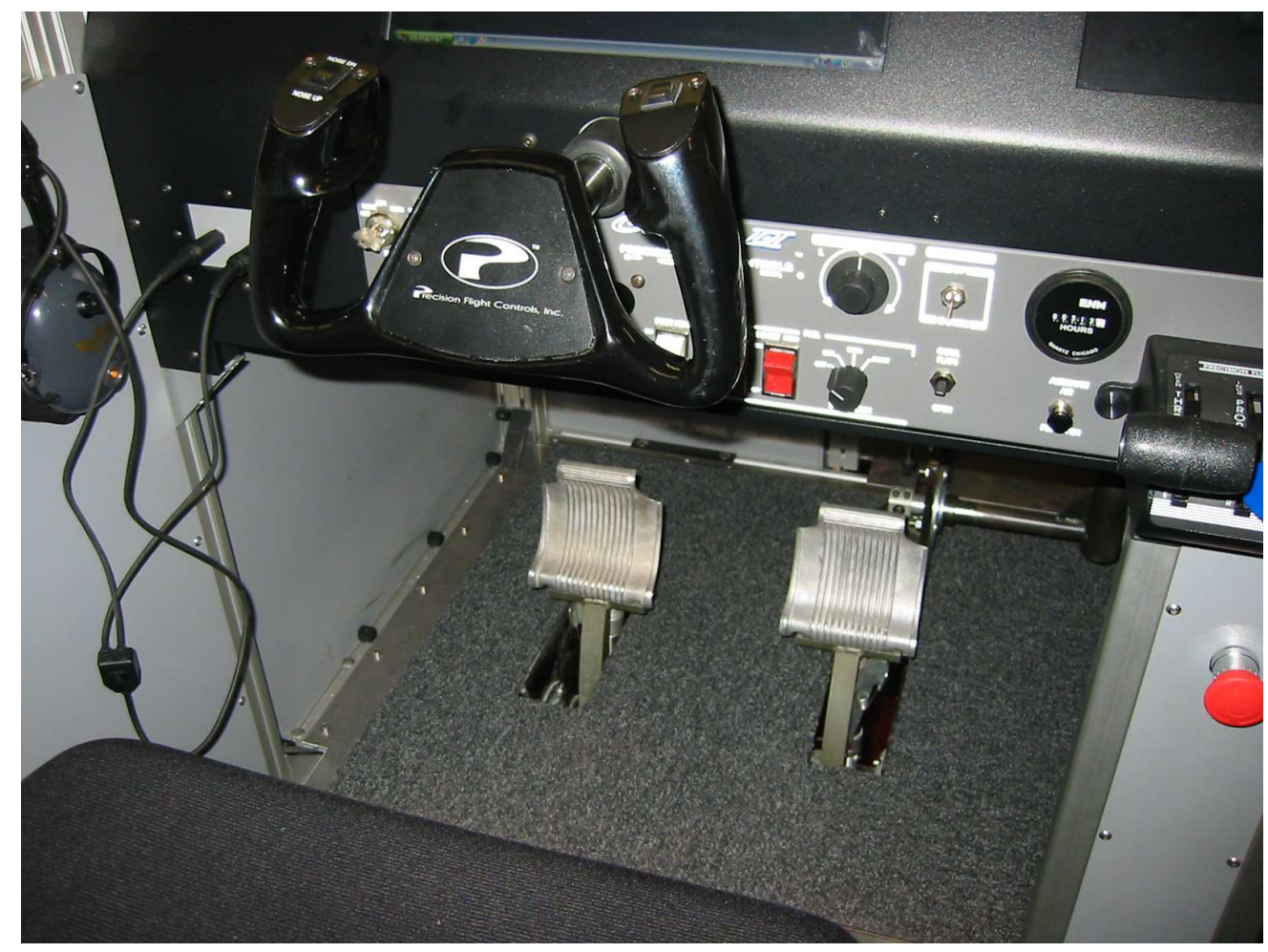

Figure 7. Flight controls

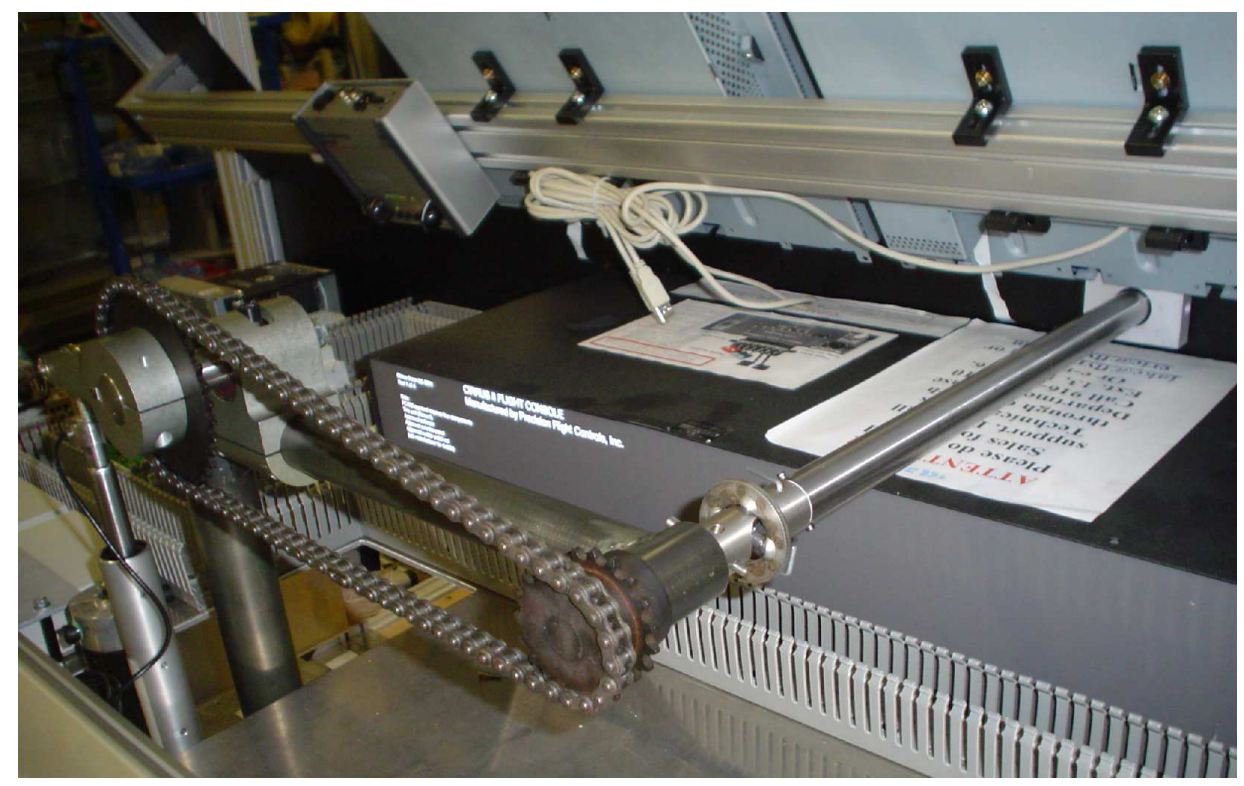

Figure 8. Detail of yoke loading leverages (back side of cockpit dashboard). An actuator is visible on the left (vertically positioned) and the yoke hub on the right 


\section{Computer and software architecture}

The overall simulator computer architecture is represented in Figure 9. The scheme reports the main flows of signals from the supervisor room computers to the rest of the simulator hardware. The layout is optimized with respect to the performance required by each piece of software used by the system. The design of the wiring, needed for hardware interconnections, has been optimized with respect to the ease of use of both car and aircraft. The external wires of the cockpits are plugged on a dedicated interface on the primary adaptor plate, Figure 10.

When the cockpits are swapped, the wires are unplugged from the formerly utilized cockpit and then plugged to the new one in use.

\section{A. Software modules}

The chosen software module that guides the various components of the system is based on FlightGear, ${ }^{7,14}$ a civilian open-source flight simulator comparable to other very popular, commercial desktop flight simulators. The version of FlightGear currently used to drive the simulator is decomposed into various modules:

- a dynamic model of the airplane; the one currently chosen is JSBSim ${ }^{15,16}$

- $P L I B$, a portable API that contains the base functions of the graphical and sound environment;

- SimGear, the main management module which sits on top of the PLIB library and controls the simulation and the scene construction;

- TerraGear, a set of tools dedicated to the terrain generation, importing GIS data, DTM, etc.

The image generation in FlightGear allows displaying both 3D and 2D. The main functions on top of classic 3D image generation software are:

- Displays of the moon, stars and sun.

- Head-up display (HUD).

- Interactive dashboard display.

- Clouds, wind

The images are generated using PC technology, by the graphic board NVIDIA GeForce Quadro FX4000. The simulation of aircraft motion, the cockpit instrument panel and flight controls, and the outside scenery are all managed by a number of instances of FlightGear talking to each other via net protocols. Moreover, the simulation is supported by two other software modules that control: $(i)$ the motion platform, in conjunction with the external view generation module, in order to give a proper acceleration feel to the user, and (ii) a force reproduction system on the cockpit controls that adds realism to the pilot's task. 


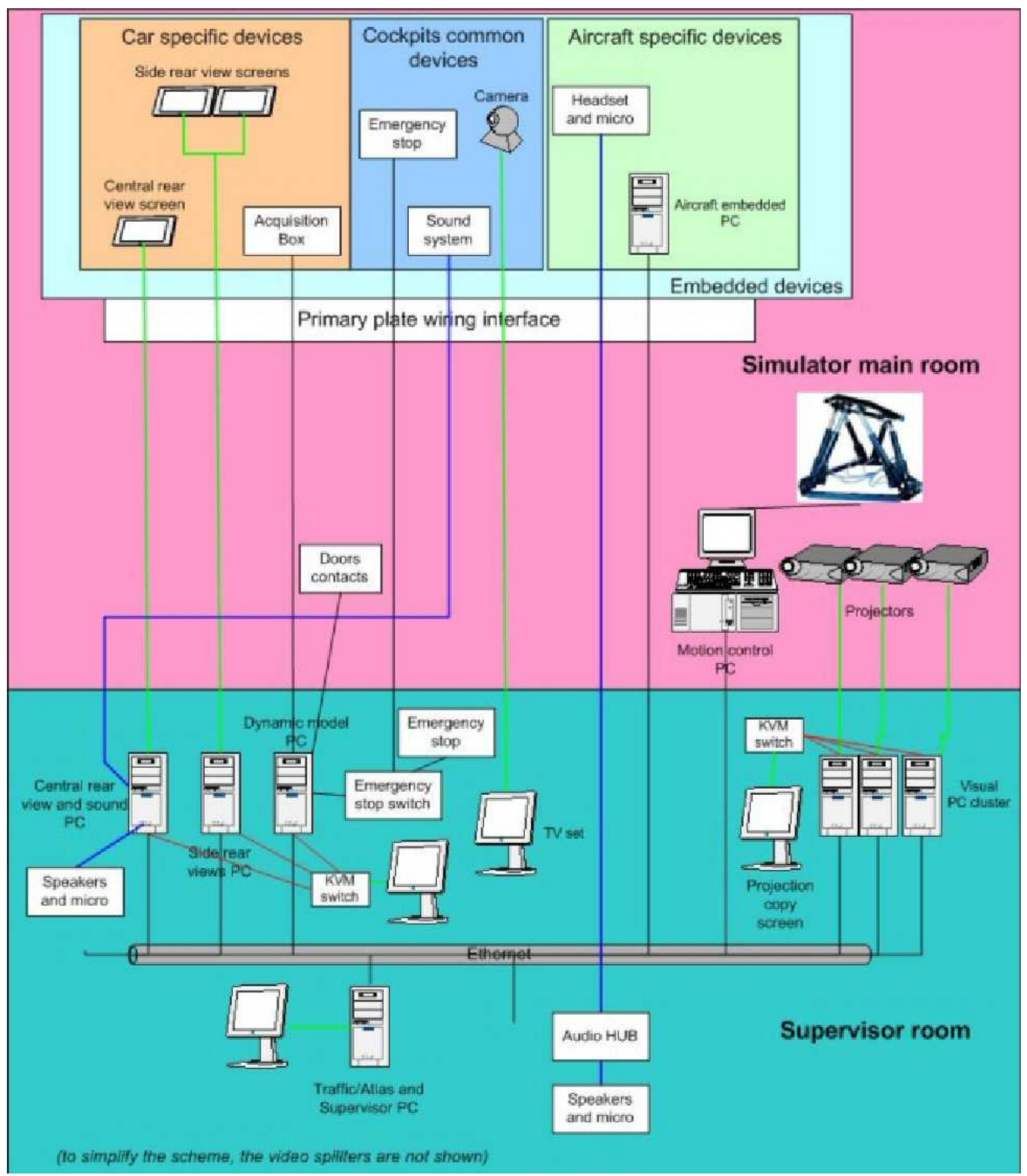

Figure 9. Scheme of hardware layout 


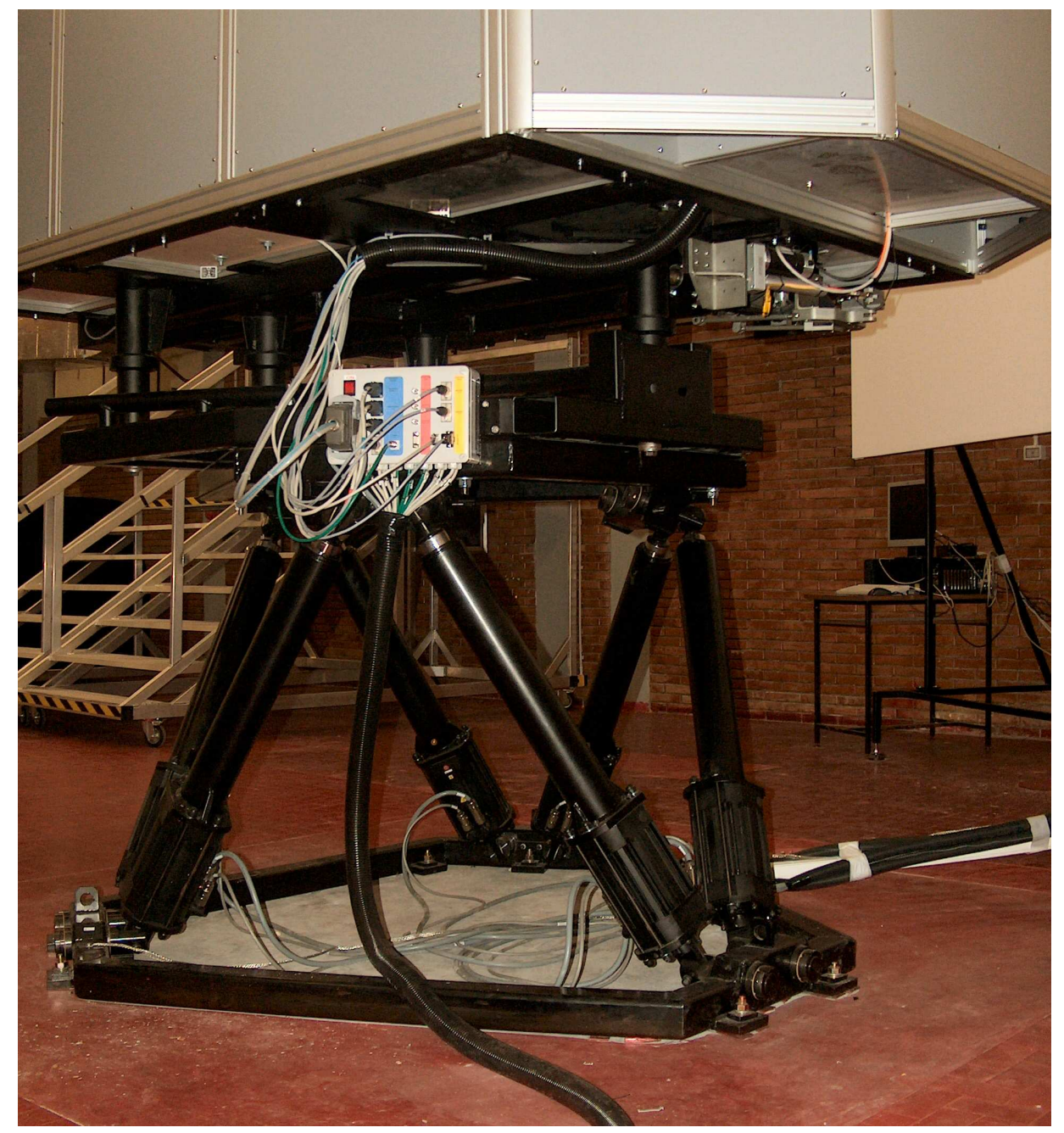

Figure 10. Interface between external wiring and cockpit internal hardware 


\section{Motion system characteristics}

The motion system is based on a six-degree-of-freedom motion base Maxcue 610-450-16-12 from cueSim, Figure 3 and Figure 10. The motion base, or motion platform, consists of a top frame, bottom frame and six high efficiency electric actuators arranged in the Stewart platform format. The payload is mounted onto the top frame.

The actuators are secured to the frames by precision manufactured pivots to provide a stiff, stable platform. Each actuator comprises a precision ball-screw directly driven by a brushless servomotor with position feedback, Figure 11.

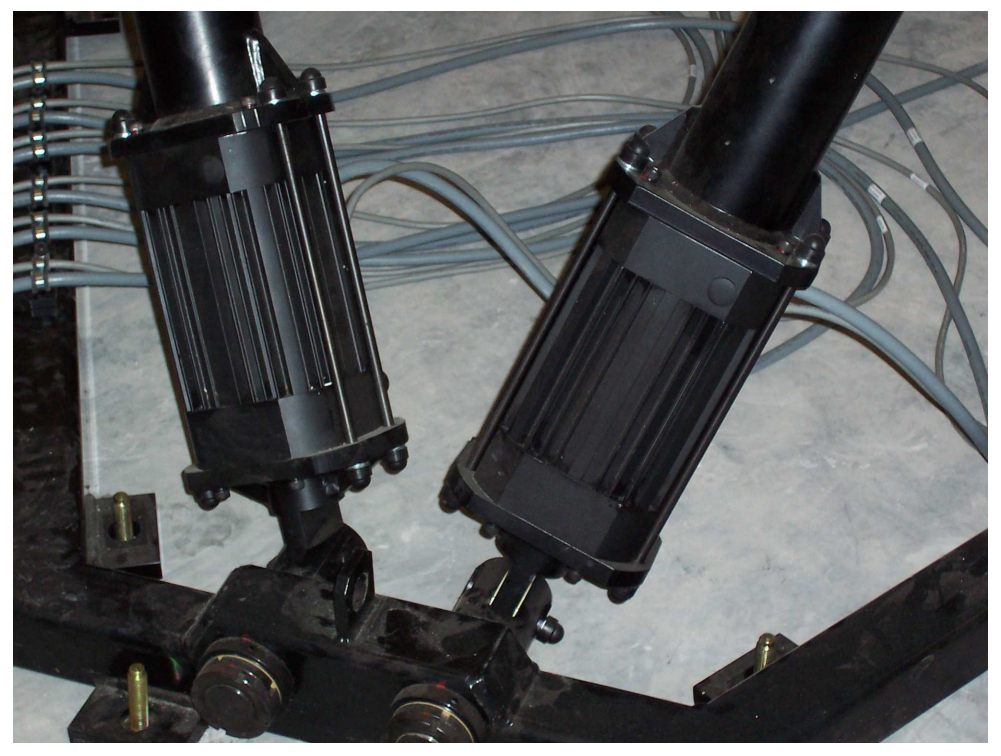

Figure 11. Motion base servomotor detail

The combination of sub-micron position resolution, high actuator peak thrust and low backlash allow high-fidelity motion with low tracking errors. The absence of belts or gearboxes in the drive system produces smooth, quiet motion. The actuators are oil lubricated and require the minimum periodic maintenance. The main characteristics of the dynamic motion base are the following:

Table 1. Motion platform characteristics

\begin{tabular}{l|c|c|c}
\hline & min/max position & peak velocity & peak acceleration \\
\hline Surge & $-491 /+432 \mathrm{~mm}$ & $718 \mathrm{~mm} / \mathrm{s}$ & $\pm 1,39 \mathrm{~g}$ \\
\hline Sway & $-425 /+425 \mathrm{~mm}$ & $712 \mathrm{~mm} / \mathrm{s}$ & $\pm 1,2 \mathrm{~g}$ \\
\hline Heave & $-247 /+248 \mathrm{~mm}$ & $484 \mathrm{~mm} / \mathrm{s}$ & $\pm 0,59 \mathrm{~g}$ \\
\hline Roll & $-25 /+25 \mathrm{deg}$ & $50 \mathrm{deg} / \mathrm{s}$ & $575 \mathrm{deg} / \mathrm{s}^{2}$ \\
\hline Pitch & $-24 /+25 \mathrm{deg}$ & $48 \mathrm{deg} / \mathrm{s}$ & $595 \mathrm{deg} / \mathrm{s}^{2}$ \\
\hline Yaw & $-43 /+43 \mathrm{deg}$ & $82 \mathrm{deg} / \mathrm{s}$ & $1100 \mathrm{deg} / \mathrm{s}^{2}$ \\
\hline
\end{tabular}

The maximum admissible payload is $1000 \mathrm{~kg}$. The actual mass of the cockpit, including that of two subjects, arrives at $500 \mathrm{~kg}$ and ensures a good level of performance of the motion platform. 


\section{Control loading reproduction}

The flight simulator is provided with a dedicated control loading module, also called force feedback module, that reproduces the piloting efforts on the yoke (push/pull, turn left/right) and on pedals, according to the simulated flight condition. The aim of the current system is to model the loading conditions on yoke and pedals of a general aviation aircraft with reversible aerodynamic control surfaces. The implementation is such that future extensions to the current model in order to simulate partial or fully powered controls are conceptually straightforward.

An overview of the control loading system is presented next.

\section{A. Model of aerosurface dynamics}

Simulation of a pilot-in-the-loop general manoeuvred flight requires the solution of the classical set of airplane equations of motions, the dynamic equations, coupled with two auxiliary sets of equations for the attitude and flight path determination, the kinematic equations.

The dynamic equations are time-stepped starting from a suitable set of initial conditions. The forcing terms in the dynamic equations are due to the effects of gravity, of aerodynamics and propulsion. They incorporate the actions due to pilot's input and, when applicable, to the ground reactions. These terms are computed at each time step and serve to advance the simulated aircraft state at the successive instant. At each time step, the two systems of auxiliary kinematic equations are then solved for the airplane attitude and mass center position update. Details on the way the classical aircraft equations of motion are coupled with auxiliary equations and solved in the time domain may be found in classical textbooks. ${ }^{10,11}$ The whole simulation proceeds in this fashion until the user stops the loop or a crash situation is detected.

The above algorithm is implemented by FlightGear's aircraft flight dynamics model. More precisely, the integration of aircraft equations of motion is performed by a sub-module of FlightGear and the user can select the desired solver to carry out the simulation. The default module, and probably the better available, is JSBSim, ${ }^{16}$ which implements a solver of aircraft non-linear equations of motion according to an advanced flight dynamics model (FDM). Both FlightGear and JSBsim are written in the $\mathrm{C}++$ programming language.

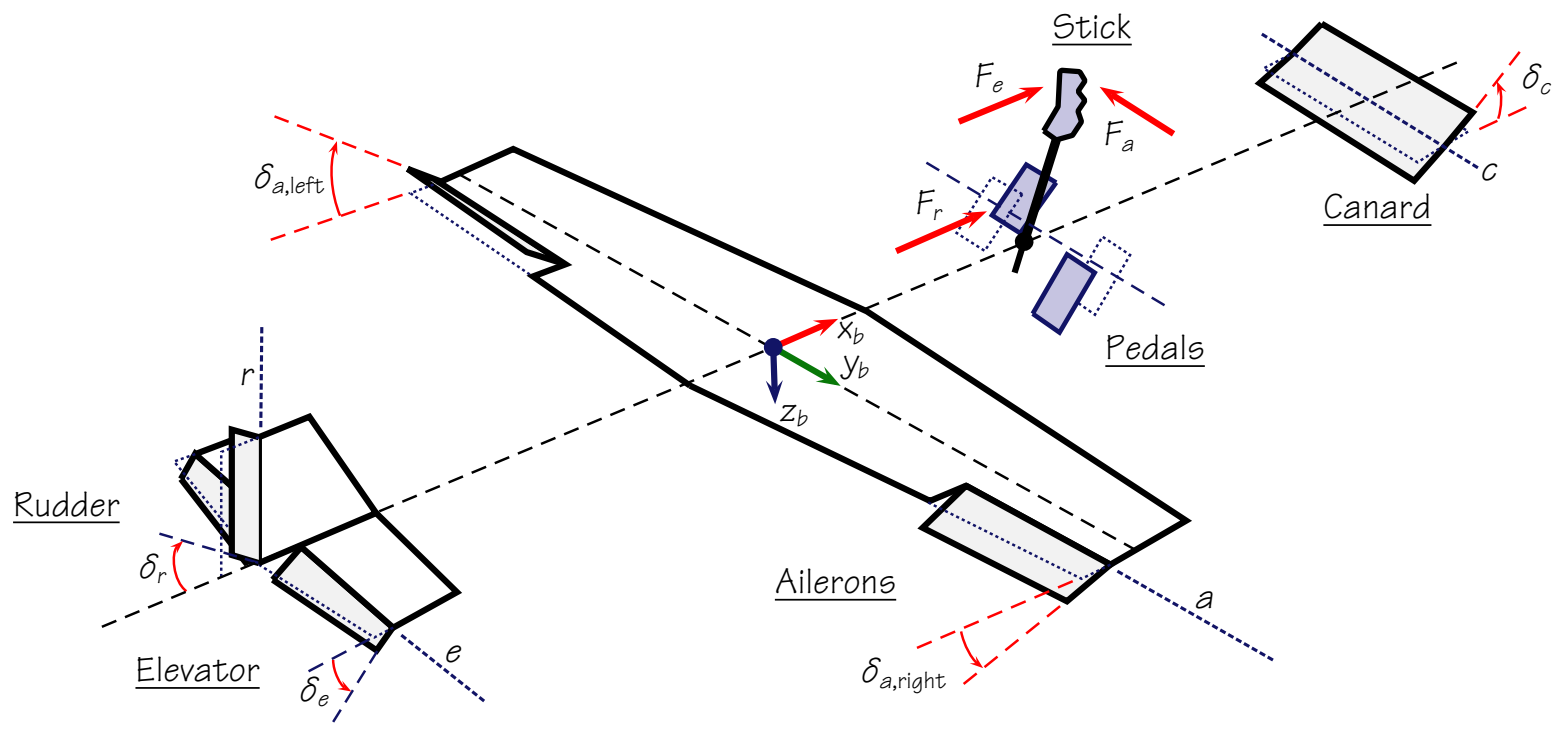

Figure 12. Sign conventions in the dynamic models of the aerodynamic surfaces. An European convention is adopted for stick forces

Following Etkin ${ }^{10}$ and Calcara, ${ }^{11}$ and selecting the conventions of Figure 12, the general equations of motion of the aerodynamic control surfaces are written as

$$
I_{a} \ddot{\delta}_{a}+2(\dot{p}+q r) I_{a y}=H_{a, \mathrm{~A}}+H_{a, \mathrm{C}}
$$


for ailerons,

$$
I_{e} \ddot{\delta}_{e}-(\dot{q}-p r) I_{e y}+\left(a_{G_{z}}-g_{z}\right) m_{e} e_{e}=H_{e, \mathrm{~A}}+H_{e, \mathrm{C}}
$$

for the elevator, and

$$
I_{r} \ddot{\delta}_{r}-(\dot{r}+p q) I_{r x}-(r q-\dot{p}) I_{r z}-\left(a_{G_{y}}-g_{y}\right) m_{r} e_{r}=H_{r, \mathrm{~A}}+H_{r, \mathrm{C}}
$$

for the rudder. A typical general aviation aircraft is represented in Figure 13 with the aim of clarifying the meaning of some quantities in equations (1), (2), (3).

- The terms $I_{a[e, r]}$ are the control surface moments of inertia about the corresponding hinge axis, $a[e, r]$.

- The quantities $a_{G_{z[y]}}$ are components onto the standard body-fixed axes $z_{b}\left[y_{b}\right]$ of the acceleration vector $\vec{a}_{G}$, acceleration of airplane's mass center $G$. The acceleration vector is given by the classical derivation formula:

$$
\vec{a}_{G}=\dot{\vec{V}}_{G}+\vec{\Omega} \wedge \vec{V}_{G}
$$

with $\vec{V}_{G}$ the velocity vector of $G$ and $\vec{\Omega}$ the instantaneous angular rate vector of the aircraft carried frame with respect to a fixed frame. When expressed in terms of scalar components in the body frame, the acceleration components are given by

$$
\left\{\begin{array}{l}
a_{G_{x}}=\dot{u}+(w q-v r) \\
a_{G_{y}}=\dot{v}+(u r-w p) \\
a_{G_{z}}=\dot{w}+(v p-u q)
\end{array}\right.
$$

with $u, w$ and $v$ the components of $\vec{V}_{G}$ onto the body-fixed axes. At simulation time the quantities on the left-hand side of equations (4) are easily accessed within those "exposed" by the flight simulation software FlightGear.

- The quantities $g_{x}, g_{y}$, and $g_{z}$ are the components of the vertical acceleration vector on the aircraft body frame. Also these are easily accessed at simulation time within those exposed by the flight simulation software.

- The eccentricities $e_{e[r]}$ in (2) and (3) are the distances between the control surface mass centers, $G_{e[r]}$, and the corresponding hinge axis. Their sign is assumed positive when the control surface center of mass lies behind the hinge axis. They result in an inertial moment about the hinge when multiplied by the control surface mass $m_{e[r]}$ and by the relevant component of the aircraft mass center acceleration $a_{G_{z[y]}}-g_{z[y]}$. When the mass centers $G_{a[e, r]}$ are projected onto the respective hinge axes the points $C_{a[e, r]}$ are obtained (see Figure 13).

- The $I_{(\cdot)(\cdot)}$ quantities with double subscripts are products of inertia. In the above formulas we have, respectively, the inertia products: of the right aileron with respect to the aileron hinge axis $a$ and the aircraft body axis $x_{b}$, i.e. $I_{a x}$; of the elevator with respect to the elevator hinge axis $e$ and the aircraft body axis $y_{b}$, i.e. $I_{e y}$; and of the rudder with respect to the hinge axis $r$ and the aircraft body axes $x_{b}$ and $z_{b}$, i.e. $I_{r x}$ and $I_{r z}$. Following Calcara, ${ }^{11}$ the typically non-zero quantities are the following

$$
\left\{\begin{array}{l}
I_{e y}=m_{e} e_{e} x_{C e}-I_{e} \cos \Lambda_{e} \\
I_{a y}=m_{a} e_{a} x_{C_{a}}-I_{a} \cos \Lambda_{a} \\
I_{r z}=m_{r} e_{r} x_{C_{r}}-I_{r} \cos \Lambda_{r}
\end{array}\right.
$$

while $I_{r x}$ is typically zero. Coordinates $x_{C_{e}}, x_{C_{a}}$ and $x_{C_{r}}$ in formulas (5) are distances, with sign, of hinge points $C_{e}, C_{a}$ and $C_{r}$ from body plane $y_{b} z_{b}$. The angles $\Lambda_{e}, \Lambda_{a}$ and $\Lambda_{r}$ are those between hinge axes $e, a$ and $r$ and body axes $y_{b}, y_{b}$ and $-z_{b}$ respectively. These quantities are shown in Figure 13.

The preceding equations reveal the requirements for dynamic balance of the control surfaces, that is, the situation in which the motion of each surface is driven only by the hinge moments $H_{\mathrm{A}}$ and $H_{\mathrm{C}}$. For instance (2) shows that, if the eccentricity of the elevator center of gravity $e_{e}$ is not zero, then an acceleration of the airplane in the $z_{b}$ direction, as in a pull-up or a banked turn, will tend to induce a torque load onto the 


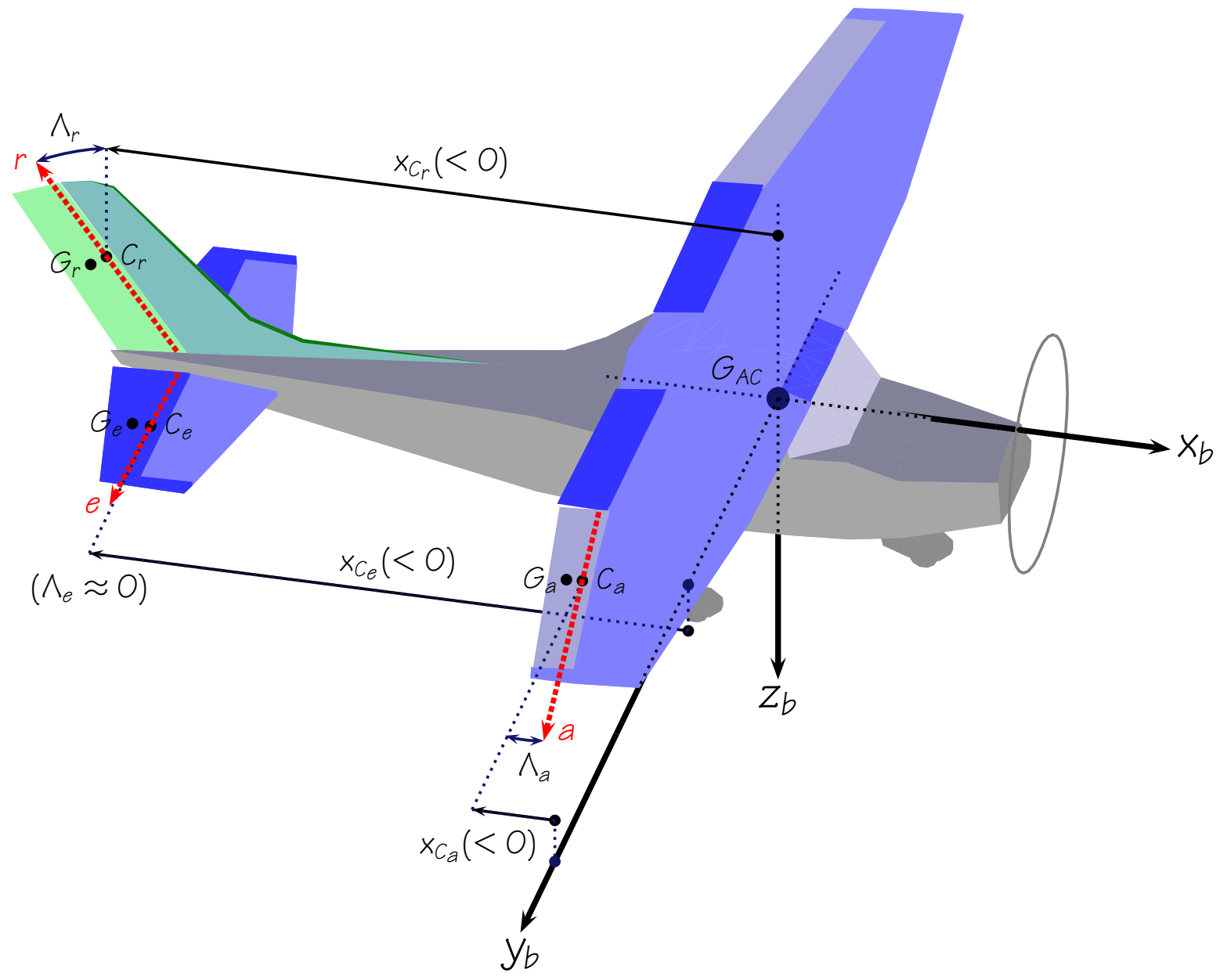

Figure 13. Definitions of frames and of significant geometric entities in the hinge moment medels

elevator. If the product of inertia $I_{e y}$ is not zero, then a roll-yaw combination $p r$, or a pitching acceleration $\dot{q}$ will tend to induce a response of the elevator. These effects are called sometimes inertial coupling effects of the elevator with the relevant airplane degrees of freedom.

Figure 14 shows the conventionally positive moments acting on a couple of traditional elevators, whose motion is a rotation about the respective hinge. The action of the pilot is the control hinge moment $H_{e, \mathrm{C}}$ ("C" stands for "commanded"). The aerodynamic action is the hinge moment $H_{e, \mathrm{~A}}$. Inertial actions consist in:

- the moment $I_{e} \ddot{\delta}_{e}$ due to the accelerated elevator rotation about the hinge,

- the moment $m_{e}\left(a_{G_{z}}-g_{z}\right) e_{e}$ due to the elevator's eccentricity,

- and in the sum of elemental terms like $d_{e} x(p r-\dot{q}) \mathrm{d} m$, where $d_{e}$ is the distance of the elemental mass $\mathrm{d} m$ from the hinge and $x$ is the coordinate of $\mathrm{d} m$ in the aircraft body frame. This sum results in the term $I_{e y}(p r-\dot{q})$ in the left hand side of (2).

The inertial coupling of a control surface is entirely eliminated when it is designed with both eccentricity and product of inertia equal to zero, then it is said dynamically balanced. When this is not the case, this inertial effect, $H_{e, \text { Inertial }}$, has to be accounted for by the model.

When the eccentricities are zero the control surface are simply said statically balanced. In practice the elevator and rudder are never dynamically balanced, even when having a swept back hinge line. The static balancing of all the control surfaces is often obtained with bob weights or by properly designing the part that lies ahead of the hinge. 


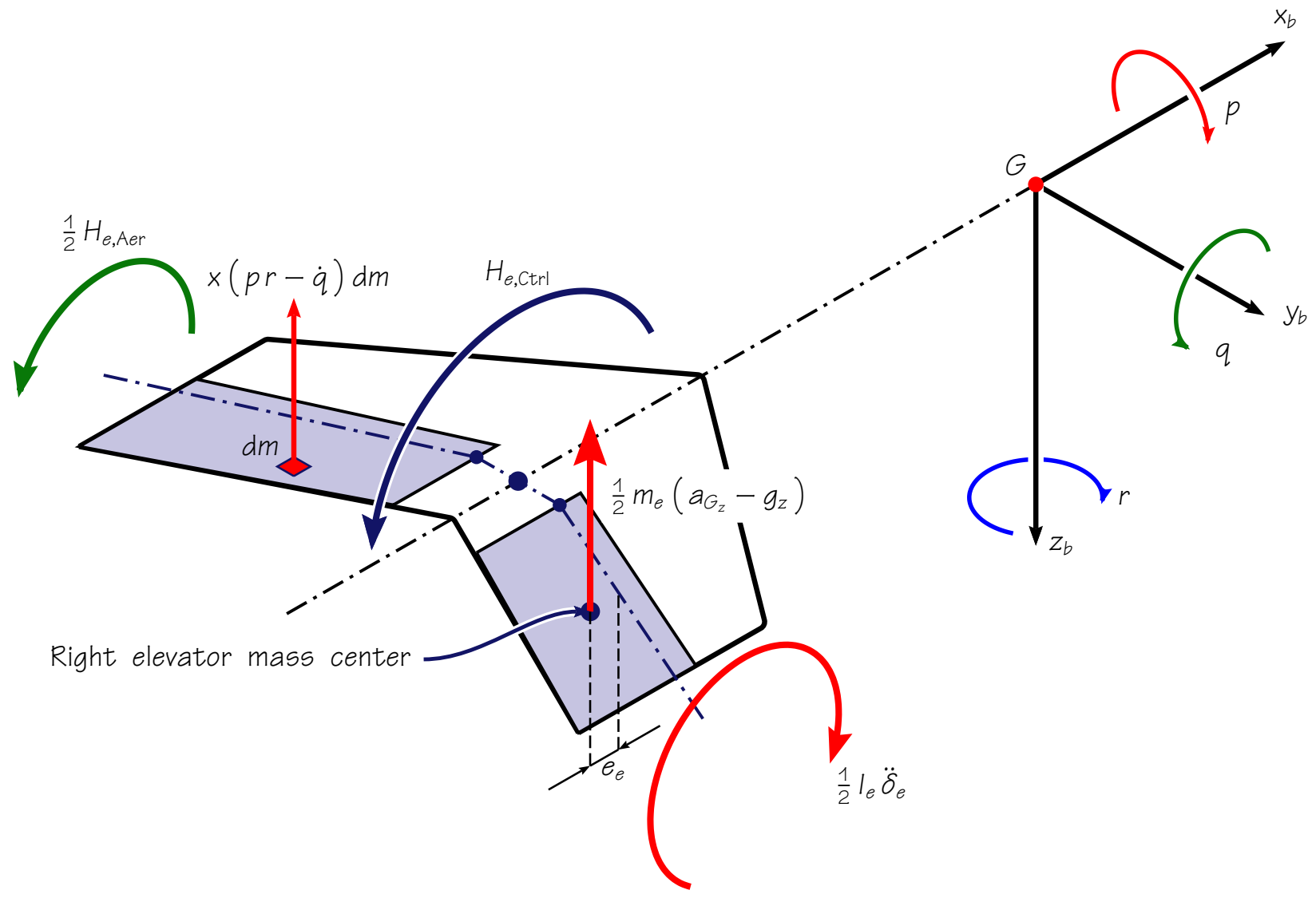

Figure 14. Schematic of the actions acting on the elevator

When a force feedback system is matched with the solver of aircraft motion equations, the actions on the aerodynamic control surfaces - which are directly transferred to the cockpit controls as column and pedal loads in a reversible system - have to be computed from the known aircraft state at each simulated time step. Stick and pedal loads are synthesized with a given frequency, typically higher than the simulation frequency, and properly reproduced (see Rolfe ${ }^{8}$ or Lee ${ }^{9}$ for a general discussion). This is mainly due to the presence of the pilot in the simulation loop and to the fact that the action of pilot on the primary controls have to be accurately measured.

In our control loading module the simulation of the aerodynamic control surfaces (ailerons, elevator, rudder) has been extended, with respect to the functionalities of FlightGear and JSBSim, and implemented in a dedicated piece of software (see ForceGear in Figure 20). The evaluation of the aerodynamic and inertial actions on the aerodynamic control surfaces is one of the main tasks of this code. It solves the equations of motion (1)-(2)-(3) between two successive FlightGear time steps. The external actions on each aerosurface are evaluated in what is also referred to as the "inner" integration loop. FlightGear's job is then called the "outer" integration loop.

At this point we recall that the stick-free conditions are those particular situations in which the pilot actions on the cockpit controls are null and the aerodynamic control surfaces are free to float under the effect of external actions. In stick-free condition terms like $F_{e, \mathrm{C}}$ in equation (2) are zero.

Aerosurface external actions depend: $(i)$ on the aircraft motion and acceleration, and (ii) on the characteristics of the mechanical linkage between the control column located in the cockpit and the tail plane moving parts. In all cases the excursions time rates are evaluated and used by the force feedback module to reproduce friction-dependent effects.

In the general case of simulated stick-free flight of a general aviation aircraft with reversible controls the angular deflections of aerodynamic surfaces represent a set of additional state variables. Then the additional unknown time histories of angular excursions $\delta_{a}(t)$ (right aileron), $\delta_{e}(t)$ (elevator or stabilator), $\delta_{r}(t)$ (rudder) have to be determined by the control surface model. 
In commanded manoeuvres the pilot control force, for instance the longitudinal effort $F_{e, \mathrm{C}}$, is non-zero and has to be treated as an input in the model equation (2). The algorithm that produces the force cue to the pilot has to go necessarily through the measurement of the action $F_{e, \mathrm{C}}$ actually exerted on the yoke by the subject pilot. Then the algorithm has to evaluate the inertial coupling and aerodynamic terms, and finally has to synthesize and reproduce the angular acceleration contained in the term $I_{e} \ddot{\delta}_{e}$. The details of this process are explained next.

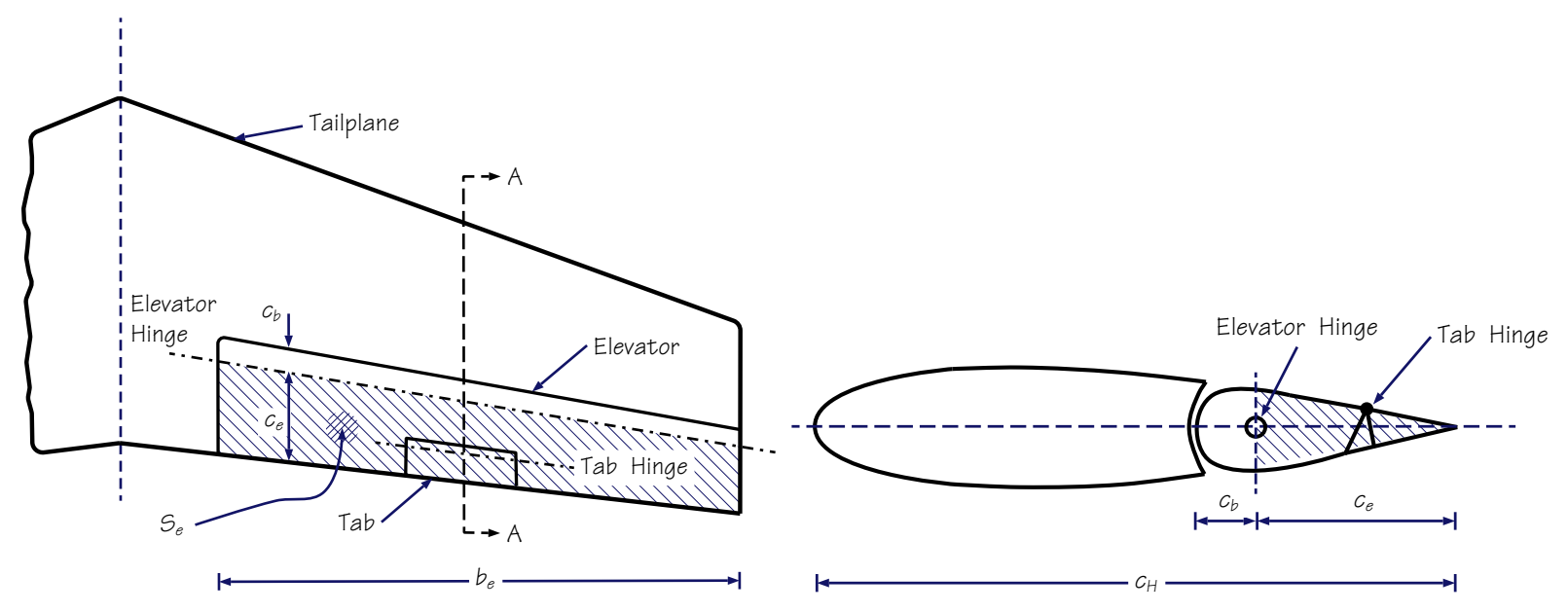

Figure 15. Tail planform and section geometries

\section{B. The control loading loop}

As anticipated in the previous section, in our flight simulator control loading module the equations (1)-(2)(3) are solved within the inner loop between two successive FlightGear time steps. The aim of the inner loop is to synthesize a movement of yoke and pedals, the feedback, which is based on aerosurface dynamics, giving the desired feel to the pilot. If pilot's action is adequate to react to the feedback and is able to keep the control position stationary, the flight conditions remain stick-fixed, or nearly so. If not, the unbalance between the force actually exerted on the control and the one calculated by the force-feedback system from simulated flight data results in a general manoeuvred flight with a varying excursion of one or possibly all the aerodynamic control surfaces.

Let us consider, for example, the case of Figure 16 where all the elements involved in longitudinal control feel reproduction are schematically represented. The key equation to be considered here is the elevator model equation (2), rewritten in the following form

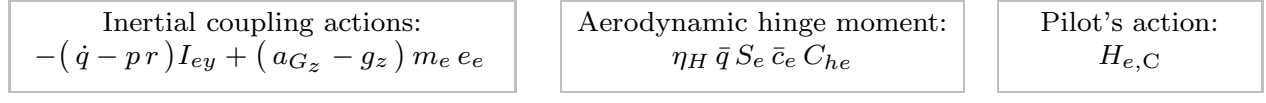

$$
\begin{aligned}
& I_{e} \ddot{\delta}_{e}+\left(-H_{\text {Inertial }}\right) \quad=\quad H_{e, \mathrm{~A}}+F_{e, \mathrm{C}} / G_{e}
\end{aligned}
$$

At the beginning of a generic inner control loop, the actual pilot effort $F_{e, \mathrm{C}}$ in equation (6) is measured by a load cell, which is part of the control loading hardware. The current stick position $s_{e}$ is sensed by a potentiometer. The quantity $s_{e}$ is directly related to the elevator position $\delta_{e}$ through the gearing ratio $G_{e}=\delta_{e} / s_{e}$. Multiple measures are performed within each inner loop iteration and treated by a Kalmann filtering procedure. Kalmann filters are typically used to eliminate noise from position and load sensors. ${ }^{19}$

The aerodynamic and inertial coupling actions are computed from the current aircraft state - i.e. from the known quantities $\dot{q}, p, r, a_{G z}, g_{z}, \bar{q}$ at the beginning of each inner loop - and from the control surface data $I_{e}, I_{e y}, m_{e}, e_{e}, \eta_{H}, S_{e}, \bar{c}_{e}, C_{h e}, G_{e}$.

The classical equilibrium condition: $G_{e} H_{e, \mathrm{~A}}=F_{e, \mathrm{C}}$, which is valid for a steady state flight along a straight path is obviously a particular case of equation (6). This situation may occur in simulation when the absolute value of the measured force on the stick equals the sum of aerodynamic and inertial coupling actions, $H_{e, \mathrm{C}}+H_{\text {Inertial. }}$. Consequently the resulting stick acceleration $\ddot{s}_{e}=\ddot{\delta}_{e} / G_{e}$ is zero. Therefore, equation (6) 


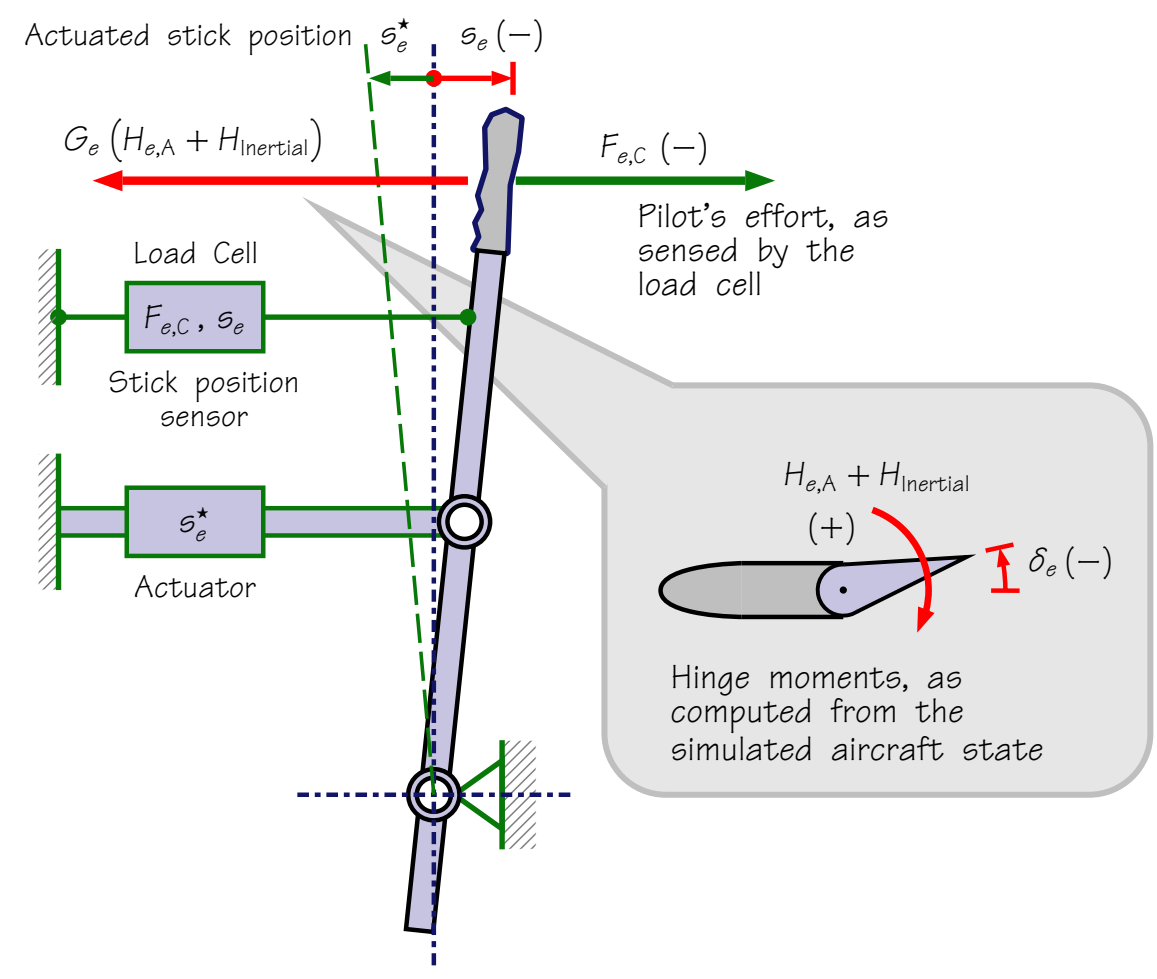

Figure 16. Schematic of longitudinal control loading

becomes

$$
G_{e}\left(H_{\text {Inertial }}+H_{e, \mathrm{~A}}\right)+F_{e, \mathrm{C}}=0
$$

The control loading module matched with the flight simulation module should be able to reproduce situations represented by (7). The control algorithm should move the stick in a position that gives the pilot the proper feel. This stimulates him to supply a force that perfectly contrasts the sum of all the remaining external actions occurring in steady flight.

In the general case of simulated manoeuvred flight the deflection $\delta_{e}(t)$ is seen as an additional state variable. In stick free conditions, $F_{e, \mathrm{C}}=0$, the unknown $\delta_{e}$ can be determined by integrating equation (6). In practice, when the control feel reproduction unit is equiped with a position actuator, the quantity of interest is $\ddot{s}_{e}$. The actuator translates a given stick acceleration (input) into a corrensponding displacement (output), according to its own inertia and response characteristics. In stick-free flight, for a stable aircraft, the resulting stick displacement tends to stabilize at the position corresponding to the equilibrium stick-free deflection $\delta_{e, \text { trim. }}$

If pilot's action is present but is not adequate to contrast the external actions, the unbalance between the force $F_{e, \mathrm{C}}$ and the term $G_{e}\left(H_{e, \mathrm{C}}+H_{\text {Inertial }}\right)$ results in the actual displacement of the control column. The displacement is obtained from the acceleration

$$
\ddot{s}_{e}=\frac{G_{e}\left(H_{\text {Inertial }}+H_{e, \mathrm{~A}}\right)+F_{e, \mathrm{C}}}{G_{e}^{2} I_{e}}
$$

resulting from (6) in a step-by-step procedure within the inner loop. A corresponding value of the stick position and a general manoeuvred flight is determined, see Figure 16.

A similar approach is valid for the remaining control surface motions. In the general case, the classical set of aircraft equations of motion is coupled with a set of three equations expressing the accelerations $\ddot{\delta}_{a}, \ddot{\delta}_{e}(t)$, $\ddot{\delta}_{r}(t)$ in terms of the known aircraft state at each simulated time. Typically the stick and pedal accelerations are also subject to a limiter algorithm for the sake of safety. For the longitudinal control, this is equivalent to the introduction of a limiter term, $K<1$, in left hand side of equation (8),

$$
G_{e}^{2} I_{e} \ddot{s}_{e}=K\left[G_{e}\left(H_{\text {Inertial }}+H_{e, \mathrm{~A}}\right)+F_{e, \mathrm{C}}\right]
$$




\section{Implementation and system details}

Two more generalizations have been added to the model described so far. The first includes the effect of the mechanical linkage dynamics on the control surface motion. This is represented by an equivalent moment mass $m_{e}^{\star}$ ideally located at a distance $d_{e}$ from the hinge. Then the moment of inertia $I_{e}$ in equations (9) and (8) becomes more generally $I_{e, \text { tot }}=I_{e}+m_{e}^{\star} d_{e}^{\star 2}$. An explanation of how this model is obtained is reported in the schematic illustration of Figure 17.

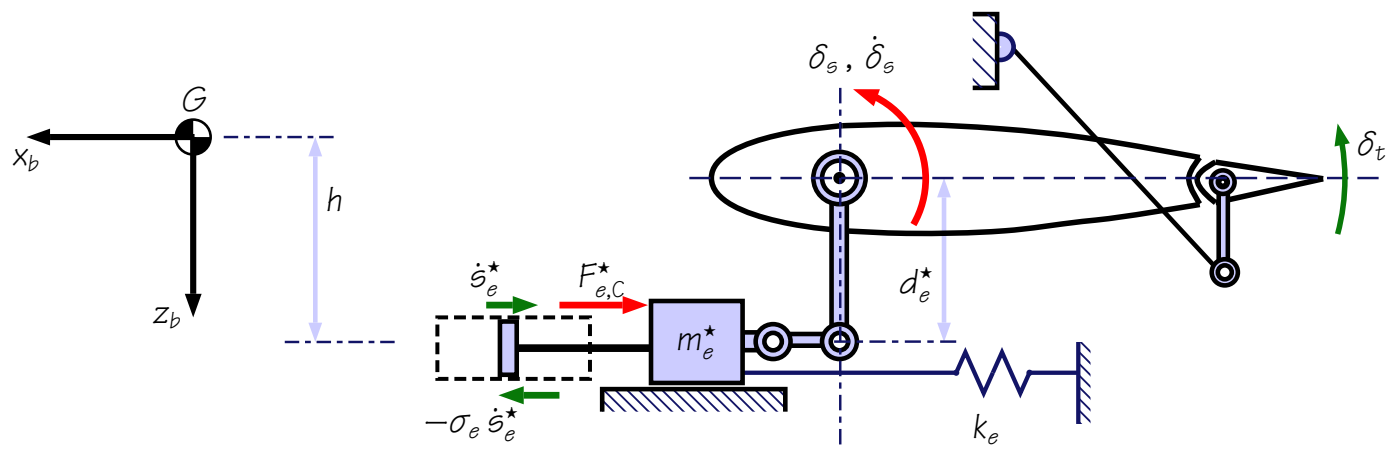

Figure 17. A reduced mass model of stabilator mechanical leverages

The second generalization includes the effects on the control displacement due to the mechanical friction (f) and to the presence of a spring (k). These are represented by two terms $F_{e, \mathrm{f}}=-\sigma_{e} \dot{s}_{e}$ and $F_{e, \mathrm{k}}=-k_{e} s_{e}$ as follows

$$
\ddot{s}_{e}=\frac{G_{e}\left(H_{\text {Inertial }}+H_{e, \mathrm{~A}}\right)+F_{e, \mathrm{C}}+F_{e, \mathrm{f}}+F_{e, \mathrm{k}}}{G_{e}^{2}\left(I_{e}+m_{e}^{\star} d_{e}^{\star^{2}}\right)}
$$

The quantity $\sigma_{e}$ is clearly a damping term whose units are $\mathrm{N} \mathrm{s} / \mathrm{m}$ and multiplies the current control displacement rate. The force feedback module calculates the displacement rate $\dot{s}_{e}$ at each cycle of the inner loop iteration.

The quantity $k_{e}$ is the stiffness of the spring in $\mathrm{N} / \mathrm{m}$ and multiplies the current control displacement. The displacement $s_{e}$ is determined by the force feedback module by evaluating the current aerodynamic surface deflection $\delta_{e}$ and applying the gearing transform: $s_{e}=\delta_{e} / G_{e}$. The latter relationship is a general result ${ }^{17}$ stating that, independently of the linkage details, there is a simple relationship between cockpit control movement and control surface excursion. For the elevator excursion $\delta_{e}$ is expressed in terms of the longitudinal stick displacement $s_{e}$ by a linear law. The dimensional quantity $G_{e}$ is referred to as the elevator control gearing ratio or, simply, the elevator gearing. It is a positive quantity having physical dimensions of $\mathrm{rad} / \mathrm{ft}$ or $\% / \mathrm{m}$. Typically the gearing may be thought of as a constant. For a specific airplane, the gearings of each control surface $G_{e}, G_{a}$, and $G_{r}$ are determined by examining the mechanical linkages between the control stick (or wheel) and both the elevator and ailerons, and between pedals and rudder.

The aircraft aerosurface data, i.e. the geometric and dynamic characteristics of each control surface and the hinge moment coefficients $C_{h, e}, C_{h, a}$ and $C_{h, r}$, are all managed by the control loading software. At each integration time step the needed coefficients, for instance the elevator hinge moment coefficient, are retrieved from a suitable database with a table look-up procedure. The entries are a number of given parameters of the motion, such as the aircraft angle of attack and airspeed. The latter are taken from one of the data structures exposed by the main simulator code via a socket protocol. This is one of the most important capabilities of FlightGear: to allow the interfacing with an external client code like our control loading software running onto a different dedicated computer.

The computer program driving the force feedback system is configured using input files in XML format. The configuration style follows the FlightGear and JSBSim philosophy. This choice guarantees a level of uniformity with the main simulator configuration format and allows a smooth transition to possible future integrations with newer releases of FlightGear. The input file format is well structured in its nature and allows an easy and clear definition of aircraft control systems and their operating characteristics, according to the particular control model implemented.

The configuration contains a description of the following items: control surface geometric and mass properties (shapes, dimensions, hinge position and orientation, eccentricities, moments of inertia), control system 
mechanical properties (gearings, friction factor, spring stiffness), control surface aerodynamic characteristics (hinge moment aerodynamic coefficients), control surface auxiliary characteristics, data logging parameters.

The actuators and the rest of the hardware of the force feedback system have been chosen in order to reproduce a realistic amount of effort required to the subject pilot. The following are the main characteristics:

- Maximum force on yoke of $\pm 400 \mathrm{~N}$ (push/pull)

- Maximum torque on yoke of $\pm 40 \mathrm{~N} \cdot \mathrm{m}$ (turn left/right)

- Maximum force on each pedal $400 \mathrm{~N}$

When needed, the force feedback can be easily disconnected.

\section{Examples of flight session with control loading}

Figures 18 and 18 show two examples of simulated manoeuvres for a Cessna C172 aircraft.

In Figure 18 the time histories start from a trimmed condition. At a given time, the pilot pulls the yoke then goes hands-off. After a while he grabs the yoke again. A closer look at the plots shows how the force feedback handles the time window in which the flight is stick-free. The quantity $F_{e, F F B}$ shown in the middle plot is the total feedback force, i.e. the numerator in right-hand side of equation (10). The force $F_{e, \mathrm{C}}$ is measured by the load cell on the yoke, i.e. is the one applied by the pilot.

In Figure 19, again, the time histories start from a trimmed condition. The pilot pushes abruptly with the right foot on the pedal then lets the pedals free to oscillate. After a while the oscillations die out naturally because of the flight dynamics model. The quantity $F_{r, \mathrm{FFB}}$ shown in the middle plot is the total feedback force on the pedal. The force $F_{r, \mathrm{C}}$ is measured by the load cell connected to the pedal linkage, i.e. is the one applied by the pilot.

As seen from the figures, in our simulation the magnitudes of these efforts are always within the range of predicted control force in steady flight at the same mean speed (see examples on McCormick's book ${ }^{17}$ for a similar aircraft).

In all figures we also show the time histories of hinge moment coefficients. These are handled by our enhancement of JSBSim's flight dynamics model. 

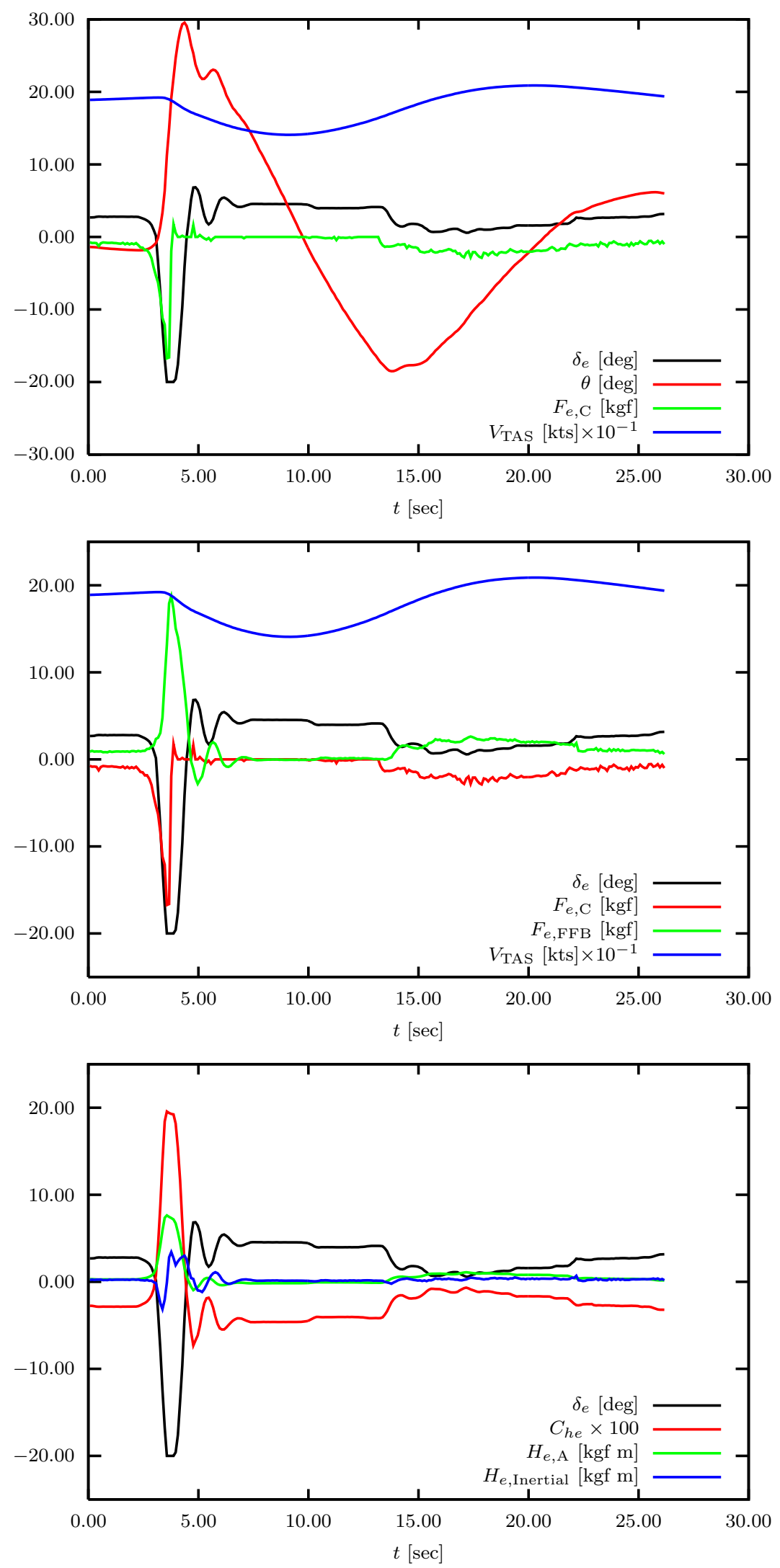

Figure 18. Example of simulated manoeuvre for a Cessna C172. From a trimmed condition the pilot pulls the yoke then goes hands-off. After a while, after $13 \mathrm{sec}$, he grabs the yoke again. The quantity $F_{e, \text { FFB }}$ shown in the second plot is the total feedback force, i.e. the numerator in right-hand side of equation (10). The force $F_{e, \mathrm{C}}$ is measured by the load cell on the yoke, i.e. is the one applied by the pilot.

\section{0 of 23}



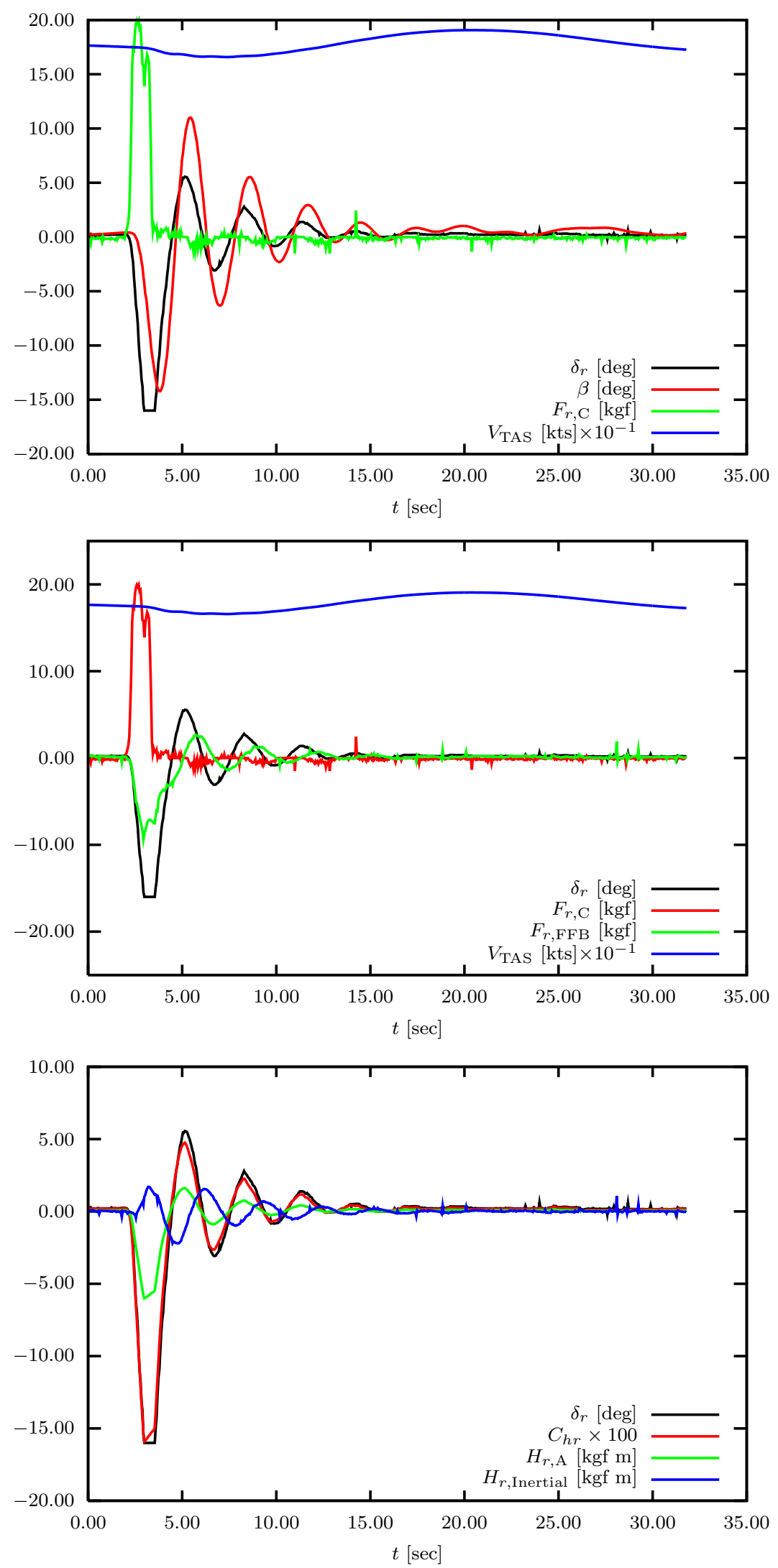

Figure 19. Example of simulated manoeuvre for a Cessna C172. From a trimmed condition the pilot pushes with the right foot on the pedal then lets the pedals free to oscillate. After a while the oscillations die out naturally because of the flight dynamics model. The quantity $F_{r}$,FFB shown in the second plot is the total feedback force on the pedal. The force $F_{r, \mathrm{C}}$ is measured by the load cell on the pedal, i.e. is the one applied by the pilot. 


\section{Current flight simulation research at UoN}

Figure 20 depicts the interconnection between the simulator operating characteristics and flight tests and simulation research topics investigated by the authors.

As shown in the top-left side of the figure, to carry out a flight simulation of a given aircraft one has to collect a number of data coming from: (i) flight tests, $(i i)$ wind tunnel experiments, (iii) numerical or semiempirical estimations. Experiences gained by the authors in these fields are reported in the works by Coiro et al., ${ }^{1-4,6}$ All aspects regarding European JAR-VLA certification procedures have been object of research. Accurate and detailed analysis of flight test maneuvers have been performed and comparisons with numerical predictions have been done. All G97 performances have been measured. ${ }^{2}$ Particular attention has also been given to the parameters estimation for the complete aircraft aerodynamic and dynamic characterization. As seen from Figure 20 (top/center), these data are properly structured in XML format according to the FlightGear configuration style.

The force feedback model is highlighted in the bottom/right part of the figure. The geometric, mass, inertia, and aerodynamic characteristics of the control surfaces along with the mechanical properties of the command transmission are taken into account in the force cue to the cockpit controls via the force feedback module. This is driven by the ForceGear software designed by the authors and implemented in cooperation with Oktal, the French company who set up the whole system.

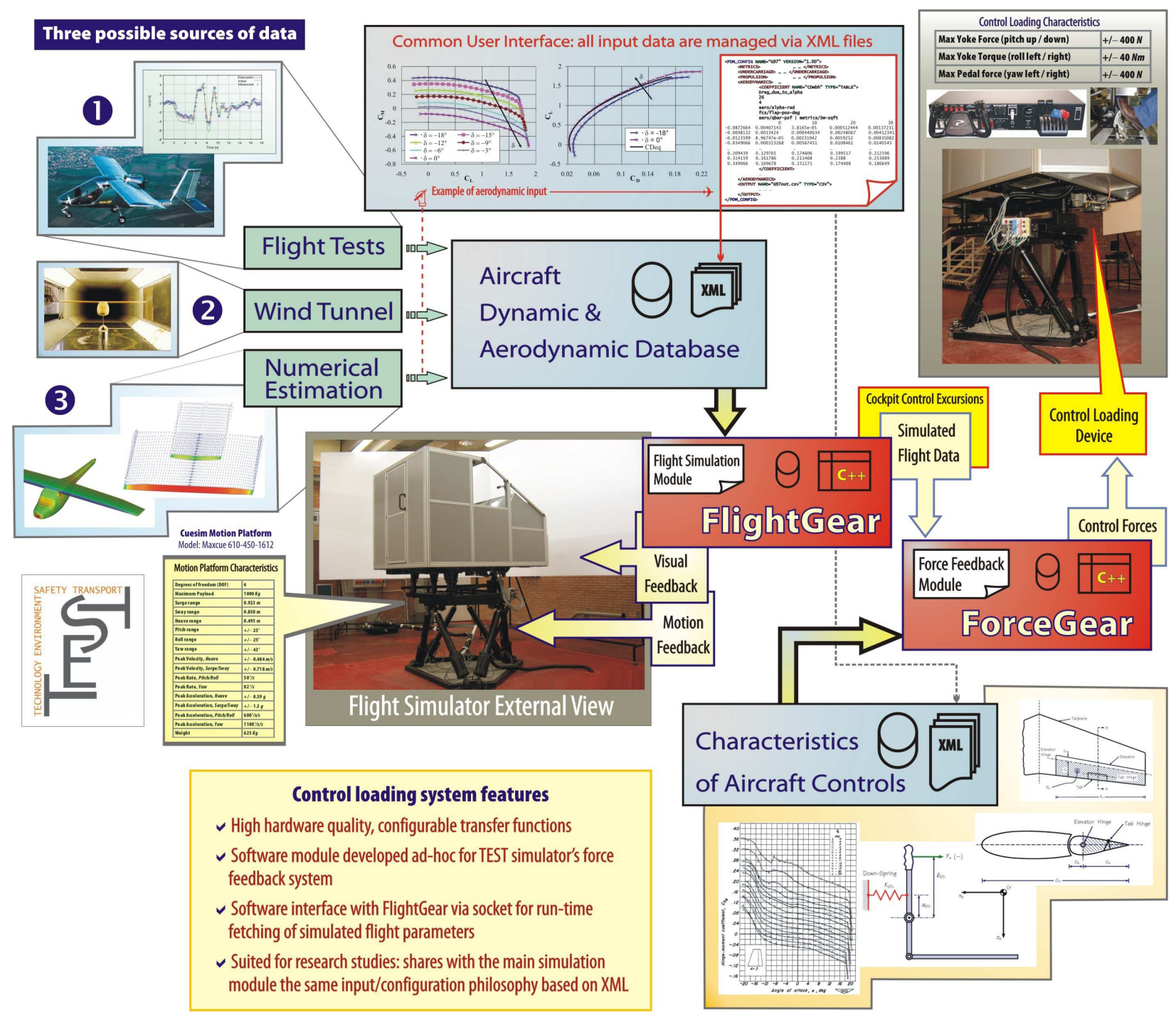

Figure 20. Flight simulation research topics investigated by the authors and their connection to the simulator characteristics 


\section{Conclusions}

The work introduced the simulation facility of the University of Naples. A unique simulation lab has been presented, i.e a simulator using the open source flight simulation software FlightGear, in conjunction with both a $6 \mathrm{DOF}$ motion base and a control loading reproduction module.

\section{References}

${ }^{1}$ Coiro, D. P.; Marulo, F.; Nicolosi, F.; Ricci, F.: "Numerical, Wind Tunnel and Flight Tests for P92J and P96 Light Aircraft." ICAS 21, Melbourne, Australia, September 1998.

${ }^{2}$ Giordano, V.; Coiro, D. P.; Nicolosi, F.: "Flight Tests, Flight Simulation and V.El. Certification on G97 Light Aircraft." XVI AIDAA National Congress, Palermo, 24-28 Settembre 2001.

${ }^{3}$ Coiro, D. P.; Nicolosi, F.; De Marco, A.: "Performances and Dynamic Behaviour Determination of DG400 Sailplane through Flight Tests." Technical Soaring, February 2002.

${ }^{4}$ Coiro, D. P.; Nicolosi, F.; De Marco, A.; Genito, N.: "Flying Qualities Analysis of A Three surfaces Aircraft Model." ICAS Conference, Toronto, Canada, September 2002.

${ }^{5}$ Coiro, D.P.: "RPV and Light Aircraft Aerodynamics, Performances and Flying Qualities Estimation, Simulation and Flight Tests." Invited lecture at: DINCON 2003, International Conference on System Identification, San Paolo, Brasil, 18-23 August 2003.

${ }^{6}$ Iscold, P.H. A. de O.; Ribeiro, R. P.; Pinto, R. L. U. de F.; Resende, L. S.; Coiro, D. P., Nicolosi, F.; Genito, N.: "Light Aircraft Instrumentation to Determine Performance, Stability and Control Characteristics in Flight Tests." SAE BRASIL Congress 2004, SAE Technical Papers Series, ISSN 0148-7191.

${ }^{7}$ Sehgal, B.; Deters, R. W. and Selig, M. S.: "Icing Encounter Flight Simulator." AIAA Paper 2002-0817. 40th AIAA Aerospace Sciences Meeting and Exhibit, Reno, NV, 2002.

${ }^{8}$ Rolfe, J. M.; Staples, K. J. (ed.s): Flight Simulation. Cambridge Aerospace Series, Cambridge University Press, 1988.

${ }^{9}$ Lee, A. T.: Flight simulation: virtual environments in aviation. Ashgate Publishing Limited, 2005.

${ }^{10}$ Etkin, B.: Dynamics of Flight, Stability and Control. John Wiley \& Sons, 1982.

${ }^{11}$ Calcara, M.: Elementi di dinamica del velivolo (Vol. I and II). Edizioni CUEN, Napoli, 1988.

${ }^{12}$ Coiro, D. P.; De Marco, A.; Leoncini P.: "Advanced Accident Flight Path Simulation and Innovative Visual Animation." Proceeding of ESMc2003 (European Simulation and Modelling Conference), Naples, October 2003.

${ }^{13}$ Coiro, D. P.; De Marco, A.: "Airplane Control Force Reproduction in Flight Simulation." Private communication 2005.

${ }^{14}$ FlightGear home page: http://www.flightgear.org/

${ }^{15}$ JSBSim home page: http://www.jsbsim.org/

${ }^{16}$ Berndt, J. S.: "JSBSim: An Open Source Flight Dynamics Model in C++." AIAA Modeling and Simulation Technology Conference, 16-19 August 16, 2004, Providence, Rhode Island.

${ }^{17}$ McCormick, B. W.: Aerodynamics, Aeronautics, and Flight Mechanics. John Wiley \& Sons, 1979.

${ }^{18}$ Etkin, B.: Dynamics of Atmospheric Flight, Dover Publications, 2005.

${ }^{19}$ Grewal, S.; Andrews A. P.: Kalmann Filtering: Theory and Practice Using Matlab. John Wiley \& Sons, Inc., 2001.

${ }^{20}$ Coiro, D. P.; De Marco, A.; Nicolosi, F.: "The Flight Simulation Environment of The University of Naples." Proceeding of ISC 2006 (International Simulation Conference), Palermo, July 2006. 